\title{
Protocol of the EFFORT study: a prospective study of FOLFIRI plus aflibercept as second- line treatment after progression on FOLFOXIRI plus bevacizumab or during maintenance treatment in patients with unresectable/metastatic colorectal cancer
}

Hironaga Satake ${ }^{1}$, Koji Ando $^{2}$, Eiji Oki ${ }^{2 *}$ D, Mototsugu Shimokawa ${ }^{3}$, Akitaka Makiyama $^{4}$, Hiroshi Saeki ${ }^{5}$, Akihito Tsuji $^{6}$ and Masaki Mori ${ }^{2}$

\begin{abstract}
Background: FOLFOXIRI plus bevacizumab is used as a first-line therapy for patients with unresectable or metastatic colorectal cancer. However, there are no clear recommendations for second-line therapy after FOLFOXIRI plus bevacizumab combination. Here, we describe our planning for the EFFORT study to investigate whether FOLFIRI plus aflibercept has efficacy following FOLFOXIRI plus bevacizumab for mCRC.

Methods: EFFORT is an open-label, multicenter, single arm phase II study to evaluate whether a FOLFIRI plus aflibercept has efficacy following FOLFOXIRI plus bevacizumab for mCRC. Patients with unresectable or metastatic colorectal cancer who received FOLFOXIRI plus bevacizumab as a first-line therapy will receive aflibercept and FOLFIRI (aflibercept $4 \mathrm{mg} / \mathrm{kg}$, irinotecan $150 \mathrm{mg} / \mathrm{m}^{2} \mathrm{IV}$ over $90 \mathrm{~min}$, with levofolinate $200 \mathrm{mg} / \mathrm{m}^{2} \mathrm{IV}$ over $2 \mathrm{~h}$, followed by fluorouracil $400 \mathrm{mg} / \mathrm{m}^{2}$ bolus and fluorouracil $2400 \mathrm{mg} / \mathrm{m}^{2}$ continuous infusion over $46 \mathrm{~h}$ ) every 2 weeks on day 1 of each cycle. The primary endpoint is progression-free survival (PFS). To achieve 80\% power to show a significant response benefit with a one-sided alpha level of 0.10 , assuming a threshold progression-free survival of 3 months and an expected value of at least 5.4 months, we estimated that 32 patients are necessary. Secondary endpoints include overall survival, overall response rate, safety, and exploratory biomarker analysis for differentiating anti-VEGF drug in 2nd-line chemotherapy for unresectable or metastatic colorectal cancer.

* Correspondence: okieiji@surg2.med.kyushu-u.ac.jp

${ }^{2}$ Department of Surgery and Science, Graduate School of Medical Science, Kyushu University, Fukuoka 812-8582, Japan

Full list of author information is available at the end of the article

(c) The Author(s). 2020 Open Access This article is licensed under a Creative Commons Attribution 4.0 International License, which permits use, sharing, adaptation, distribution and reproduction in any medium or format, as long as you give appropriate credit to the original author(s) and the source, provide a link to the Creative Commons licence, and indicate if changes were made. The images or other third party material in this article are included in the article's Creative Commons licence, unless indicated otherwise in a credit line to the material. If material is not included in the article's Creative Commons licence and your intended use is not permitted by statutory regulation or exceeds the permitted use, you will need to obtain permission directly from the copyright holder. To view a copy of this licence, visit http://creativecommons.org/licenses/by/4.0/ The Creative Commons Public Domain Dedication waiver (http://creativecommons.org/publicdomain/zero/1.0/) applies to the data made available in this article, unless otherwise stated in a credit line to the data. 
(Continued from previous page)

Discussion: This is the first study to investigate whether FOLFIRI plus aflibercept has efficacy following FOLFOXIRI plus bevacizumab for unresectable or metastatic colorectal cancer. Switching to a different type of anti-VEGF drug in second-line therapy after FOLFOXIRI plus bevacizumab appears to be an attractive treatment strategy when considering survival benefit. It is expected that this phase II study will prove the efficacy of this strategy and that a biomarker for drug selection will be discovered.

Trial registration: Japan Registry of Clinical Trials jRCTs071190003. Registered April 18, 2019.

Keywords: Colorectal cancer, Aflibercept, FOLFIRI, Second line, FOLFOXIRI, Bevacizumab, Anti-VEGF drug

\section{Background}

One of the goals in chemotherapy for unresectable/ metastatic colorectal cancer (mCRC) is to prolong survival and maintain quality of life by controlling the disease through exposure to all active agents in an appropriate sequence of administration. Nine different classes of drugs have shown antitumor activity in mCRC: fluoropyrimidines, irinotecan, oxaliplatin, anti-epidermal growth factor receptor (EGFR) antibodies, anti-vascular endothelial growth factor (VEGF) drugs, regorafenib, trifluridine/tipiracil (FTD/TPI, TAS-102), encorafenib for $B R A F$ V600E mutant $\mathrm{mCRC}$, and immunotherapy for microsatellite instability-high/deficient mismatch repair (MSI-H/dMMR) mCRC. Chemotherapy is usually performed with a combination of cytotoxic drugs and a molecular target drug such as anti-VEGF drug or antiEGFR antibody. A cytotoxic DOUBLET combination of fluorouracil (5-FU) plus levofolinate (l-LV) and either oxaliplatin (FOLFOX) or irinotecan (FOLFIRI) with a molecular target drug is generally proposed as initial systemic chemotherapy; recently, however, a TRIPLET combination of fluorouracil plus levofolinate, oxaliplatin and irinotecan (FOLFOXIRI) showed superior efficacy in terms of tumor shrinkage and survival benefit compared with the DOUBLET combination.

The TRIBE study showed that FOLFOXIRI plus bevacizumab $(\mathrm{BEV})$ is a promising regimen in first-line therapy for patients with $\mathrm{mCRC}$ [1], and this regimen is now regarded as a recommended first-line therapy for patients whose treatment goal is tumor shrinkage and in patients with $B R A F$ mutant tumors. However, a secondline therapy after FOLFOXIRI plus BEV treatment has not been well established. The TRIBE2 study showed that after maintenance treatment with 5-FU/ 1-LV plus $\mathrm{BEV}$, re-introduction of FOLFOXIRI plus BEV offered the most favorable survival benefit [2]. However, most patients who receive an oxaliplatin-based regimen experience peripheral sensory neuropathy. Therefore, FOLFIRI plus BEV appears to be the most commonly used regimen for second-line therapy after FOLFOXIRI plus BEV [1]. Although FOLFIRI plus BEV may be suitable as a standard regimen for second-line therapy, all of the drugs in this regimen are included in first-line
FOLFOXIRI plus BEV; accordingly, a response to FOLFIRI plus BEV would not be expected following the failure of first-line FOLFOXIRI plus BEV. Recently, two new anti-VEGF drugs - aflibercept [3] and ramucirumab [4] - showed promising anti-tumor effects as second-line treatment when combined with a FOLFIRI-based regimen for patients with mCRC. FOLFOXIRI plus BEV, or its maintenance phase - 5-FU/ 1-LV plus BEV, does not include aflibercept, and thus this drug might provide additional benefit to patients who have progressed after FOLFOXIRI plus BEV.

To investigate this possibility, we planned a phase II EFFORT study to investigate whether FOLFIRI plus aflibercept has efficacy following FOLFOXIRI plus BEV treatment. Here, we describe the protocol for the phase II EFFORT study.

\section{Methods/design \\ Study design and treatment}

The EFFORT study is an open-label, multicenter, single arm phase II study to evaluate whether FOLFIRI plus aflibercept has efficacy following FOLFOXIRI plus BEV for $\mathrm{mCRC}$ in patients with unresectable or metastatic colorectal cancer. The study has been approved by a central review board and is currently ongoing at 47 medical facilities in Japan. The main inclusion criteria are histologically confirmed advanced mCRC, known $R A S$ mutation status (known $B R A F$ mutation status also, if possible), mCRC treated with FOLFOXIRI plus BEV as first-line therapy for at least two courses, adjuvant chemotherapy and FOLFOXIRI plus BEV treatment for recurrence, age $\geq 20$ years, ECOG PS 0 or 1 , measurable lesions based on the Response Evaluation Criteria in Solid Tumors (RECIST) guidelines version 1.1, adequate organ function, and sufficient oral ingestion function. Complete inclusion and exclusion criteria are shown in Table 1. RAS and BRAF testing are performed locally.

Patients receive aflibercept and FOLFIRI (aflibercept 4 $\mathrm{mg} / \mathrm{kg}$, irinotecan $150 \mathrm{mg} / \mathrm{m}^{2}$ IV over $90 \mathrm{~min}$, with $\mathrm{l}-\mathrm{LV}$ $200 \mathrm{mg} / \mathrm{m}^{2}$ IV over $2 \mathrm{~h}$, followed by 5 -FU $400 \mathrm{mg} / \mathrm{m}^{2}$ bolus and 5 -FU $2400 \mathrm{mg} / \mathrm{m}^{2}$ continuous infusion over $46 \mathrm{~h}$ ) every 2 weeks on day 1 of each cycle. Patients receive premedication with antiemetic agents according to 
Table 1 Patient inclusion and exclusion criteria

Inclusion criteria

1. Personal written informed consent is obtained after the study has been fully explained

2. The lead investigator deems that the patient can be treated according to the protocol (the patient is suitable for enrollment)

3. Histologically confirmed colon or rectal adenocarcinoma

4. RAS mutation analysis at enrollment identifies RAS status as either the wild type or mutant type

5. Patients with unresectable CRC or mCRC who received FOLFOXIRI plus bevacizumab as first-line therapy for at least two courses. First-line therapy is discontinued due to progressive disease (PD)

a. Patients with unresectable CRC or mCRC who discontinued first-line therapy with FOLFOXIRI plus bevacizumab

b. Patients who underwent adjuvant chemotherapy and FOLFOXIRI plus bevacizumab treatment following recurrence (the date of recurrence confirmation should be at least 6 months from the final day of adjuvant therapy.)

c. Patients who discontinued 5-FU/LV plus bevacizumab as a maintenance therapy (FOLFOXIRI plus bevacizumab as induction therapy will be administered for no more than 12 cycles.)

7. Age $\geq 20$ years at enrollment

8. ECOG performance status (PS) score of 0 or 1

9. Measurable lesion in accordance with RECIST ver. 1.1 criteria on contrast-enhanced chest, abdominal, or pelvic (trunk) CT (required within 28 days of enrollment)

(Measurable lesions should be $10 \mathrm{~mm}$ or more on the major axis using a CT scan with a $5 \mathrm{~mm}$ slice. For metastatic lymph nodes, the minor axis should exceed $15 \mathrm{~mm}$ in length.)

10. Patients with sufficient oral ingestion function

11. Vital organ functions meet the following criteria within 14 days before enrollment.

If multiple test results are available in that period, the results closest to enrollment is used. No blood transfusions or hematopoietic factor administration is permitted within 2 weeks before the date on which measurements are taken.

a. White blood cell count: $\geq 3000 / \mathrm{mm}^{3}, \leq 12,000 / \mathrm{mm}^{3}$

b. Neutrophil count: $\geq 1500 / \mathrm{mm}^{3}$

c. Platelet count: $\geq 7.5 \times 104 / \mathrm{mm}^{3}$

d. Hemoglobin concentration: $\geq 9.0 \mathrm{~g} / \mathrm{dL}$

e. Total bilirubin: $\leq 1.5 \mathrm{mg} / \mathrm{dL}$

f. AST, ALT: $\leq 100 \mathrm{IU} / \mathrm{L}$ ( $\leq 200 \mathrm{IU} / \mathrm{L}$ for liver metastases)

g. Serum creatinine: $\leq 1.5 \mathrm{mg} / \mathrm{dL}$, or creatinine clearance: $\geq 50 \mathrm{~mL} / \mathrm{min}$

h. Urine protein: $\leq 1+(1+$ or $<1.0 \mathrm{~g} / 24 \mathrm{~h})$

12. Life expectancy $\geq 3$ months

13. UGT1A1 polymorphism is wild type or single heterozygous type

14. Radiation therapy was not administered to the target lesion. However, patients can be included if they:

a. Have received neoadjuvant or adjuvant radiation therapy.

b. Have received radiation therapy against non-target lesions.

Exclusion criteria

1. Patients with hypertension (> $160 \mathrm{mmHg}$ systolic or $>100 \mathrm{mmHg}$ diastolic for $>4$ weeks) that cannot be adequately controlled with 2 antihypertensive agents*

*One antihypertensive treatment containing two antihypertensive agents counts as two antihypertensives.

2. Patients with diabetes mellitus that cannot be adequately controlled with medication.

3. Patients with heart disease that may cause problems during the conduct of the study, such as congestive heart failure, angina pectoris requiring medication, clear evidence of transmural myocardial infarction on ECG, clinically evident valvular heart disease, symptomatic coronary disease, poorly controlled arrhythmia, and a previous history of myocardial infarction within the last 12 months.

4. Patients with severe pulmonary disease, including interstitial pneumonia, pulmonary fibrosis and severe emphysema.

5. Patients with an active infection.

6. Patients with clinically significant ascites and pleural effusion.

7. Patients who have severe drug hypersensitivity (particularly to 5-FU, irinotecan, or aflibercept).

8. Patients with active multiple cancers. Lesions consistent with carcinoma in situ or intramucosal carcinoma that have been cured by local treatment are not classified as active multiple cancers.

9. Patients with a psychiatric disorder that may pose a problem, or a history of central nervous system dysfunction.

10. Patients who have brain metastases. 
Table 1 Patient inclusion and exclusion criteria (Continued)

\author{
11. Patients who have had a gastrointestinal perforation and/or a gastrointestinal fistula up to 6 months prior to enrollment. \\ 12. Watery diarrhea or diarrhea Grade $\geq 2$ at the time of enrollment. \\ 13. Patients who have had deep vein thrombosis, pulmonary embolism, or some other major form of thromboembolism (portal vein or catheter \\ thrombosis and superficial venous thrombosis qualify as major forms) up to 3 months prior to enrollment. \\ 14. The patient has experienced any arterial thromboembolic events, including but not limited to myocardial infarction, transient ischemic attack, \\ cerebrovascular accident, or unstable angina, within 6 months prior to the first dose of protocol therapy. \\ 15. Daily treatment with high-dose aspirin ( $\geq 325 \mathrm{mg} /$ day). \\ 16. Non-steroidal anti-inflammatory medications and immune suppressive or steroidal medications. \\ 17. Patients receiving phenytoin, warfarin potassium, or flucytosine. \\ 18. Women who are pregnant, breast feeding, or who wish to conceive. \\ 19. Men who wish to conceive. \\ 20. Patients with active gastrointestinal tract bleeding requiring repeated transfusions. \\ 21. Patients who underwent resection after FOLFOXIRI+bevacizumab because of conversion and experienced disease progression. \\ 22. Patients who are unable to tolerate aflibercept, 5-FU or irinotecan. \\ 23. Patients with a severe stenosis due to primary CRC. However, primary patients with CRC resection or colostomy can be included. \\ 24. Patients with hepatic cirrhosis or active hepatitis. \\ 25. Patients whom a lead investigator or primary physician deems are not appropriate for this study.
}

*One antihypertensive treatment containing two antihypertensive agents counts as two antihypertensives

institutional guidelines. Treatment continues until disease progression, unacceptable toxicity, death, patient refusal, or investigator decision. When irinotecan is stopped due to severe diarrhea or other adverse events, irinotecan can be skipped, in which case 5-FU/l-LV plus aflibercept or aflibercept alone can be administered. When aflibercept is missed due to an adverse event, FOLFIRI, irinotecan alone, or 5-FU/l-LV can be administered, and such treatments are also within the protocol treatment. There is no prescribed treatment following completion or discontinuation of protocol treatment. Planned enrollment period is 2019 April to 2021 March, and the observation period will include a 2 -year followup period from the time the last patient is enrolled. No interim analysis of this study will be performed.

\section{Endpoints and assessments}

The primary objective of this trial is to determine whether the FOLFIRI plus aflibercept regimen has efficacy following FOLFOXIRI plus BEV in patients with $\mathrm{mCRC}$ in terms of progression-free survival (PFS). Secondary endpoints are overall response rate (ORR), overall survival (OS) and safety.

Disease assessment is performed every 6 weeks by computed tomography $(\mathrm{CT})$. Response is determined by CT scanning based on Response Evaluation Criteria in Solid Tumors version 1.1. PFS is defined as the time from study enrollment to first disease progression or death, whichever occurs first; OS is defined as the time from study enrollment to the date of death due to any cause; and ORR is defined as the percentage of patients relative to the total of enrolled subjects who achieve a complete response (CR) or partial response (PR) based on CT scan images.

All adverse events (AEs) observed during the study treatment period are appropriately registered in the subjects' medical records and in electronic case reports forms. All serious adverse events (SAEs), namely fatal or life-threatening adverse events or those requiring hospitalization or resulting in persistence or significant disability/incapacity, are required to be disclosed by the investigator to the KSCC (Kyushu Study group of Clinical Cancer) Study Office/Enrollment and Data Analysis Center within $24 \mathrm{~h}$. AEs will be assessed according to the Common Terminology Criteria for Adverse Events version 5.0.

Plasma levels of placental growth factor (PlGF), vascular endothelial growth factor-A (VEGF-A), vascular endothelial growth factor-B (VEGF-B), vascular endothelial growth factor-C (VEGF-C), vascular endothelial growth factor-D (VEGF-D), and interleukin-8 (IL-8) are assessed in blood samples collected from each patient before the protocol treatment, prior to first imaging evaluation, and within 30 days after treatment discontinuation to identify biomarkers that predict the efficacy of aflibercept. This analysis aims to identify a potential predictive biomarker for the efficacy of FOLFIRI plus aflibercept treatment.

\section{Target sample size and statistical analyses}

The primary endpoint of this study is PFS. Second-line PFS with a FOLFIRI-based regimen after FOLFOXIRI/ $\mathrm{BEV}$ is considered to be shorter than second-line PFS after FOLFOX/BEV due to treatment resistance to 
irinotecan. According to the TRIBE-2 trial, the secondline PFS of FOLFIRI/BEV after FOLFOX/BEV was 5.6 months [2]. Furthermore, a phase 2 trial of FOLFIRI plus aflibercept conducted in Japan showed its PFS as 5.4 months (95\% CI, 4.14-6.70) [5]. Based on these results, the expected PFS value in this study was set at 5.4 months. To achieve $80 \%$ power to show a significant response benefit with a one-sided alpha level of 0.10 , and assuming a threshold PFS of 3 months, we estimated that 32 patients would be necessary. Considering dropouts, a total of 35 patients would need to be enrolled.

The following hypothesis will be tested using the confidence intervals for median survival time as defined by Brookmeyer and Crowley. Sample size calculation was performed using SAS ver.9.4 (Cary, NC, USA).

\section{Discussion}

A survival benefit for anti-EGFR antibody in 2nd-line chemotherapy has not been shown even in the case of $R A S$ wild-type mCRC [6-9]. In contrast, the combination of an anti-VEGF drug and doublet combination has shown a survival effect $[3,4,10,11]$, and an antiVEGF drug is therefore usually selected in combination with second-line chemotherapy for mCRC. While bevacizumab, aflibercept and ramucirumab are currently used as anti-VEGF drugs [3, 4, 12], no biomarker or definite criteria for selection of these drugs is available, and no data for second-line therapy after FOLFOXIRI plus $\mathrm{BEV}$ as pretreatment have been reported.

Attempts to discover a molecular predictive biomarker for anti-VEGF drugs have not led to clinically useful findings, although several studies are currently underway. The acquisition of resistance to BEV in patients with mCRC may involve BEV-induced cytokine changes and high VEGF-A, $-\mathrm{D}$ and placental growth factor (PIGF) serum levels [13-16]. Following biomarker analysis of the RAISE trial (NCT01183780), ramucirumab is likely to be added to second-line FOLFIRI for patients with high VEGF-D levels. However, ramucirumab has been reported to show negative effects when administered to patients with low VEGF-D levels equivalent to the one-third of normal levels seen after first-line treatment with a bevacizumab-containing oxaliplatin-based regimen for mCRC [17]. In contrast, a biomarker post hoc analysis of the VELOUR trial (NCT00561470) reported that aflibercept retains its activity regardless of baseline VEGF-A and PIGF levels [18]. The GI-SCREEN CRC-Ukit study, a prospective longitudinal study to investigate an association between plasma angiogenesisrelated mediators and clinical outcomes in $\mathrm{MCRC}$, suggested that changes in VEGF-D and PIGF occurred independently, and it may be possible to select drugs by assessing these alterations [19].

Furthermore, regarding the effects of these anti-VEGF drugs, the usefulness of second-line treatment after administration of FOLFOXIRI plus BEV as pretreatment has not been reported. Moreover, the desirability of switching to a different type of anti-VEGF drug in subsequent treatment following BEV as pretreatment remains unclear.

FOLFOXIRI plus BEV showed significant superiority to DOUBLET plus BEV as initial systemic chemotherapy for patients with $\mathrm{mCRC}$ in terms of survival benefit and tumor shrinkage $[1,2,20]$. Subgroup analyses also indicated that FOLFOXIRI plus BEV is remarkably effective in patients with poor prognosis, such as those with BRAF mutations, extrahepatic metastases or a rightsided primary $[1,20]$. Patients with $\mathrm{mCRC}$ who receive FOLFOXRI plus BEV as initial systemic chemotherapy may therefore expect an aggressive therapeutic combination as a subsequent regimen. Aflibercept uniquely targets both VEGF-A and PlGF, with higher affinity for both than other anti-angiogenic therapies, and VEGF-A and PlGF bind aflibercept with higher affinity than their

Table 2 Background of second-line anti-VEGF drug trials

\begin{tabular}{|c|c|c|c|c|c|c|}
\hline Trial & \multicolumn{2}{|c|}{ ML18147 Ref. [11] } & \multicolumn{2}{|c|}{ RAISE Ref. [4] } & \multicolumn{2}{|c|}{ VELOUR Ref. [3] } \\
\hline Number of cases & \multicolumn{2}{|l|}{820} & \multicolumn{2}{|l|}{1072} & \multicolumn{2}{|l|}{1226} \\
\hline Prior oxaliplatin & \multicolumn{2}{|l|}{$41.8 \%$} & \multicolumn{2}{|l|}{$100 \%$} & \multicolumn{2}{|l|}{$100 \%$} \\
\hline Prior bevacizumab & \multicolumn{2}{|l|}{$100 \%$} & \multicolumn{2}{|l|}{$100 \%$} & \multicolumn{2}{|l|}{$30.5 \%$} \\
\hline Backbone chemotherapy & \multicolumn{2}{|c|}{ Irinotecan (35\%) } & \multicolumn{2}{|l|}{ FOLFIRI } & \multicolumn{2}{|l|}{ FOLFIRI } \\
\hline Design & \multicolumn{2}{|c|}{ Open label } & \multicolumn{2}{|c|}{ Double blind } & \multicolumn{2}{|c|}{ Double blind } \\
\hline Combination & With BEV & Without BEV & With RAM & Without RAM & With AFL & Without AFL \\
\hline ORR & $5 \%$ & $3 \%$ & $13.4 \%$ & $12.5 \%$ & $19.8 \%$ & $11.1 \%$ \\
\hline \multirow[t]{2}{*}{ PFS (months) } & 5.7 & 4.1 & 5.7 & 4.5 & 6.90 & 4.67 \\
\hline & HR 0.68 & $p<.0001$ & HR0.793 & $p<.0005$ & HR0.758 & $p<.0001$ \\
\hline \multirow[t]{2}{*}{ OS (months) } & 11.2 & 9.8 & 13.3 & 11.7 & 13.50 & 12.06 \\
\hline & HR 0.81 & $p=.0062$ & HR 0.844 & $p=.0219$ & HR 0.817 & $p=.0032$ \\
\hline
\end{tabular}

ORR overall response rate, PFS progression-free survival, $O S$ overall survival, $B E V$ bevacizumab, $R A M$ ramucirumab, $A F L$ aflibercept 
native receptor [21]. These findings suggest that tumors progressing under blockade of a single anti-angiogenic therapy, such as BEV, most likely use numerous nonVEGF-A mechanisms to sustain their growth. Switching to a different therapy to target these alternative mechanisms, such as aflibercept, may be beneficial. Although differences in study design and patient characteristics hamper decision-making from cross-trial comparisons, aflibercept plus FOLFIRI combination showed an attractive ORR and survival benefit for patients with mCRC compared to BEV and ramucirumab as secondline therapy (Table 2). This study uses comparison with historical controls to investigate biomarkers able to predict the effects of aflibercept, and to determine the desirability of switching to a different type of anti-VEGF drug for second-line therapy following FOLFOXIRI therapy as pretreatment.

\section{Abbreviations}

5-FU: 5-Fluorouracil; AE: Adverse event; BEV: Bevacizumab; CRC: Colorectal cancer; CT: Computed tomography; ECOG: Eastern Cooperative Oncology Group; EGFR: Epidermal growth factor receptor; FOLFIRI: 5-

fluorouracil+levofolinate calcium+irinotecan; FOLFOX: 5fluorouracil+levofolinate calcium+oxaliplatin; FOLFOXIRI: 5-

fluorouracil+levofolinate calcium+oxaliplatin+irinotecan; IRI: irinotecan; ILV: Levofolinate calcium; mCRC: Unresectable/metastatic colorectal cancer; NCCN: National Comprehensive Cancer Network; ORR: Overall response rate; OS: Overall survival; OX: Oxaliplatin; PD: Progressive disease; PFS: Progressionfree survival; PS: Performance status; VEGF: Vascular endothelial growth factor

\section{Acknowledgements}

This trial is supported by KSCC Study Office/Enrollment and Data Analysis Center.

Editorial assistance/English editing was provided by Guy Harris of DMC Corp.

\section{Authors' contributions}

$\mathrm{EO}$ and $\mathrm{KA}$ are the principal investigators, and are responsible for the trial design and study procedures. HS, KA EO AM and HS form the Protocol Committee. HS is responsible for recruitment and patient information. MS is responsible for statistical analysis. HS, EO KA, MS, AT and MM drafted and revised the manuscript. All authors reviewed and approved the final manuscript.

\section{Funding}

The EFFORT study is being funded by Sanofi Pharmaceutical Co., Ltd. The sponsor had no control over the interpretation, writing, or publication of this paper.

\section{Availability of data and materials}

Not applicable.

\section{Ethics approval and consent to participate}

This study is being conducted in accordance with the Good Clinical Practice guidelines and the guiding principles detailed in the Declaration of Helsinki, and in keeping with applicable local law(s) and regulation(s). Written informed consent to study procedures must be provided by all candidate patients before enrolment. We have registered this study in the Japan Registry of Clinical Trials with registration number jRCTs071190003. Before initiation, the principal investigator was required to consult the Certified Review Board (Kyushu University Certified Institutional Review Board for Clinical Trials: Certification No. CRB718005) and receive approval from the study site's manager, and to submit a trial plan to the Minister of Health, Labor and Welfare.

\section{Consent for publication}

Not applicable.

\section{Competing interests}

The EFFORT study is being funded by Sanofi Pharmaceutical Co., Ltd. Hironaga Satake receives honoraria from Sanofi Co., Ltd. and Yakult Honsha Co., Ltd.

Eiji Oki receives honoraria from Yakult Honsha Co., Ltd.

All remaining authors have declared no conflict of interest.

\section{Author details}

${ }^{1}$ Cancer Treatment Center, Kansai Medical University Hospital, Hirakata 573-1191, Japan. ${ }^{2}$ Department of Surgery and Science, Graduate School of Medical Science, Kyushu University, Fukuoka 812-8582, Japan. ${ }^{3}$ Department of Biostatistics, Yamaguchi University Graduate School of Medicine, Ube 755-0046, Japan. ${ }^{4}$ Cancer Center, Gifu University Hospital, Gifu 501-1194, Japan. ${ }^{5}$ Department of General Surgical Science Graduate School of Medicine, Gunma University, Maebashi 371-8511, Japan. ${ }^{6}$ Department of Clinical Oncology, Faculty of Medicine, Kagawa University, Miki-cho 540-0006, Japan.

Received: 21 July 2020 Accepted: 26 October 2020

Published online: 17 November 2020

\section{References}

1. Loupakis F, Cremolini C, Masi G, Lonardi S, Zagonel V, Salvatore L, Cortesi E, Tomasello G, Ronzoni M, Spadi R, et al. Initial therapy with FOLFOXIRI and bevacizumab for metastatic colorectal cancer. N Engl J Med. 2014;371(17): 1609-18.

2. Cremolini C, Antoniotti C, Rossini D, Lonardi S, Loupakis F, Pietrantonio F, Bordonaro R, Latiano TP, Tamburini E, Santini D, et al. Upfront FOLFOXIRI plus bevacizumab and reintroduction after progression versus mFOLFOX6 plus bevacizumab followed by FOLFIRI plus bevacizumab in the treatment of patients with metastatic colorectal cancer (TRIBE2): a multicentre, openlabel, phase 3, randomised, controlled trial. Lancet Oncol. 2020;21(4):497507.

3. Van Cutsem E, Tabernero J, Lakomy R, Prenen H, Prausova J, Macarulla T, Ruff $P$, van Hazel GA, Moiseyenko V, Ferry D, et al. Addition of aflibercept to fluorouracil, leucovorin, and irinotecan improves survival in a phase III randomized trial in patients with metastatic colorectal cancer previously treated with an oxaliplatin-based regimen. J Clin Oncol. 2012;30(28):3499506.

4. Tabernero J, Yoshino T, Cohn AL, Obermannova R, Bodoky G, GarciaCarbonero R, Ciuleanu TE, Portnoy DC, Van Cutsem E, Grothey A, et al. Ramucirumab versus placebo in combination with second-line FOLFIRI in patients with metastatic colorectal carcinoma that progressed during or after first-line therapy with bevacizumab, oxaliplatin, and a fluoropyrimidine (RAISE): a randomised, double-blind, multicentre, phase 3 study. Lancet Oncol. 2015:16(5):499-508.

5. Denda T, Sakai D, Hamaguchi T, Sugimoto N, Ura T, Yamazaki K, Fujii H, Kajiwara T, Nakajima TE, Takahashi S, et al. Phase II trial of aflibercept with FOLFIRI as a second-line treatment for Japanese patients with metastatic colorectal cancer. Cancer Sci. 2019;110(3):1032-43.

6. Cunningham D, Humblet Y, Siena S, Khayat D, Bleiberg H, Santoro A, Bets D, Mueser M, Harstrick A, Verslype C, et al. Cetuximab monotherapy and cetuximab plus irinotecan in irinotecan-refractory metastatic colorectal cancer. N Engl J Med. 2004;351(4):337-45.

7. Hecht JR, Cohn A, Dakhil S, Saleh M, Piperdi B, Cline-Burkhardt M, Tian Y, Go WY. SPIRITT: a randomized, multicenter, phase II study of Panitumumab with FOLFIRI and Bevacizumab with FOLFIRI as second-line treatment in patients with Unresectable wild type KRAS metastatic colorectal Cancer. Clin Colorectal Cancer. 2015;14(2):72-80

8. Peeters M, Price TJ, Cervantes A, Sobrero AF, Ducreux M, Hotko Y, Andre T, Chan E, Lordick F, Punt CJ, et al. Randomized phase III study of panitumumab with fluorouracil, leucovorin, and irinotecan (FOLFIRI) compared with FOLFIRI alone as second-line treatment in patients with metastatic colorectal cancer. J Clin Oncol. 2010;28(31):4706-13.

9. Sobrero AF, Maurel J, Fehrenbacher L, Scheithauer W, Abubakr YA, Lutz MP, Vega-Villegas ME, Eng C, Steinhauer EU, Prausova J, et al. EPIC: phase III trial of cetuximab plus irinotecan after fluoropyrimidine and oxaliplatin failure in patients with metastatic colorectal cancer. J Clin Oncol. 2008;26(14):2311-9.

10. Giantonio BJ, Catalano PJ, Meropol NJ, O'Dwyer PJ, Mitchell EP, Alberts SR, Schwartz MA, Benson AB 3rd. Eastern cooperative oncology group study E: Bevacizumab in combination with oxaliplatin, fluorouracil, and leucovorin 
(FOLFOX4) for previously treated metastatic colorectal cancer: results from the eastern cooperative oncology group study E3200. J Clin Oncol. 2007; 25(12):1539-44.

11. Kubicka S, Greil R, Andre T, Bennouna J, Sastre J, Van Cutsem E, von Moos R, Osterlund P, Reyes-Rivera I, Muller T, et al. Bevacizumab plus chemotherapy continued beyond first progression in patients with metastatic colorectal cancer previously treated with bevacizumab plus chemotherapy: ML18147 study KRAS subgroup findings. Ann Oncol. 2013;24(9):2342-9.

12. Bennouna J, Sastre J, Arnold D, Osterlund P, Greil R, Van Cutsem E, von Moos R, Vieitez JM, Bouche O, Borg C, et al. Continuation of bevacizumab after first progression in metastatic colorectal cancer (ML18147): a randomised phase 3 trial. Lancet Oncol. 2013;14(1):29-37.

13. Alidzanovic L, Starlinger P, Schauer D, Maier T, Feldman A, Buchberger $E_{r}$ Stift J, Koeck U, Pop L, Gruenberger B, et al. The VEGF rise in blood of bevacizumab patients is not based on tumor escape but a host-blockade of VEGF clearance. Oncotarget. 2016;7(35):57197-212.

14. Kopetz S, Hoff PM, Morris JS, Wolff RA, Eng C, Glover KY, Adinin R, Overman MJ, Valero V, Wen S, et al. Phase II trial of infusional fluorouracil, irinotecan, and bevacizumab for metastatic colorectal cancer: efficacy and circulating angiogenic biomarkers associated with therapeutic resistance. J Clin Oncol. 2010;28(3):453-9.

15. Loupakis F, Cremolini C, Fioravanti A, Orlandi P, Salvatore L, Masi G, Di Desidero T, Canu B, Schirripa M, Frumento P, et al. Pharmacodynamic and pharmacogenetic angiogenesis-related markers of first-line FOLFOXIRI plus bevacizumab schedule in metastatic colorectal cancer. Br J Cancer. 2011; 104(8):1262-9.

16. Willett CG, Duda DG, di Tomaso E, Boucher Y, Ancukiewicz M, Sahani DV, Lahdenranta J, Chung DC, Fischman AJ, Lauwers GY, et al. Efficacy, safety, and biomarkers of neoadjuvant bevacizumab, radiation therapy, and fluorouracil in rectal cancer: a multidisciplinary phase II study. J Clin Oncol. 2009;27(18):3020-6.

17. Tabernero J, Hozak RR, Yoshino T, Cohn AL, Obermannova R, Bodoky G, Garcia-Carbonero R, Ciuleanu TE, Portnoy DC, Prausova J, et al. Analysis of angiogenesis biomarkers for ramucirumab efficacy in patients with metastatic colorectal cancer from RAISE, a global, randomized, double-blind, phase III study. Ann Oncol. 2018;29(3):602-9.

18. Van Cutsem E, Paccard C, Chiron M, Tabernero J. Impact of prior Bevacizumab treatment on VEGF-A and PIGF levels and outcome following second-line Aflibercept treatment: biomarker post hoc analysis of the VELOUR trial. Clin Cancer Res. 2020;26(3):717-25

19. Taniguchi H, Yuki S, Shiozawa M, Masuishi T, Nishina T, Kagawa Y, Takahashi $\mathrm{N}$, Yasui H, Denda T, Sunakawa Y, et al. Plasma VEGF-D and PIGF levels according to prior use of biologics among metastatic colorectal cancer: preliminary results from Gl-SCREEN CRC-Ukit study. J Clin Oncol. 2020;38(no. 4_suppl):178.

20. Cremolini C, Loupakis F, Antoniotti C, Lupi C, Sensi E, Lonardi S, Mezi S, Tomasello G, Ronzoni M, Zaniboni A, et al. FOLFOXIRI plus bevacizumab versus FOLFIRI plus bevacizumab as first-line treatment of patients with metastatic colorectal cancer: updated overall survival and molecular subgroup analyses of the open-label, phase 3 TRIBE study. Lancet Oncol. 2015;16(13):1306-15.

21. Papadopoulos N, Martin J, Ruan Q, Rafique A, Rosconi MP, Shi E, Pyles EA, Yancopoulos GD, Stahl N, Wiegand SJ. Binding and neutralization of vascular endothelial growth factor (VEGF) and related ligands by VEGF trap, ranibizumab and bevacizumab. Angiogenesis. 2012;15(2):171-85.

\section{Publisher's Note}

Springer Nature remains neutral with regard to jurisdictional claims in published maps and institutional affiliations.

Ready to submit your research? Choose BMC and benefit from:
- fast, convenient online submission
- thorough peer review by experienced researchers in your field
- rapid publication on acceptance
- support for research data, including large and complex data types
- gold Open Access which fosters wider collaboration and increased citations
- maximum visibility for your research: over 100M website views per year
At BMC, research is always in progress.
Learn more biomedcentral.com/submissions

